\title{
Direct Observations of Rotationally Distorted Stars
}

\author{
Gerard T. van Belle \\ Michelson Science Center, California Institute of Technology, 770 South \\ Wilson Ave MS 100-22, Pasadena, CA 91125
}

David R. Ciardi

University of Florida, Department of Astronomy, 211 Bryant Space

Sciences, Gainesville, FL 32611

Robert R. Thompson

Jet Propulsion Laboratory,California Institute of Technology, 4800 Oak Grove Drive MS 171-113, Pasadena, CA 91109

\author{
Rachel L. Akeson \\ Michelson Science Center, California Institute of Technology, 770 South \\ Wilson Ave MS 100-22, Pasadena, CA 91125
}

\begin{abstract}
We present infrared interferometric angular size measurements for rapidly rotating stars which indicate non-circular projected disk brightness distributions. For the A7IV-V star Altair, assuming that the apparent oblateness of the photosphere is due to the star's rapid rotation, a rigorous evaluation of the observation data in the context of a rigidly rotating Roche model shows that an estimate of $v \sin i=210 \pm 13 \mathrm{~km} \mathrm{~s}^{-1}$ can be derived that is independent of spectroscopic techniques. Altair is the first main sequence star for which direct observations of an oblate photosphere have been reported, and the first star for which $v \sin i$ has been established from observations of the star's photospheric geometry. Future prospects for this technique are considered, and a prospective catalog of 67 rotationally oblate targets is presented.
\end{abstract}

\section{Introduction}

Stellar rotation has been measured observationally for almost a century, beginning with Schlesinger $(1909,1911)$. Up until now, however, virtually all observational evidence underpinning the theoretical models has been based upon velocities inferred from spectroscopic line broadening. While this technique is both well understood and well developed, it is susceptible to confusion with other influences upon spectral line widths, such as various turbulence mechanisms and latitude dependencies of line emission (see Carpenter et al. 1984 and references therein). An independent means by which to determine the parameters governing the structure of centrifugally-distorted stars would be welcome. 
Direct observation of the stellar disk can provide unique insight into basic stellar parameters, including rotation velocity. The measured angular size in conjunction with the bolometric flux and distance yields constraints on parameters such as effective temperature and linear radii, both of which remain quantities poorly established empirically for virtually all stars. Upon fitting a family of rotating models for the projected stellar photosphere upon the sky, we further demonstrate that unique values for true rotation velocity $v$ and inclination $i$ may be derived from the interferometric data.

Our pilot study of this approach to determination of rotation velocity involved interferometric observations of the star Altair, using the Palomar Testbed Interferometer (PTI), and resulted in a geometric determination of the apparent rotation velocity $v \sin i$ (van Belle et al. 2001). The star Altair ( $\alpha$ Aql, HR 7557, HD 187642) is a well-studied object, being the 12th brightest star in the sky and one of the 50 nearest stars to the Sun (Allen 1973, Perryman et al. 1997). It is an A7IV-V main sequence star (Johnson \& Morgan 1953) and is known to be a rapid rotator, with an atmosphere that has been extensively modeled (eg. Gouttebroze et al. 1999). The measurements of the star's apparent rotational velocity $(v \sin i)$ range from $190 \mathrm{~km} \mathrm{~s}^{-1}$ (Carpenter et al. 1984) up to $250 \mathrm{~km} \mathrm{~s}^{-1}$ (Stoeckley 1968), depending upon the spectral lines used in the investigation. These values of $v \sin i$ are a substantial fraction of the star's estimated critical velocity of $430 \mathrm{~km} \mathrm{~s}^{-1}$ (Gray 1976), where centripetal acceleration at the stellar equator exceeds gravitational acceleration.

The $\sim 3$ milliarcsecond (mas) angular diameter of Altair was observed thirty years ago by Hanbury Brown and his colleagues with the Intensity Interferometer at Narrabri (Hanbury Brown et al. 1967, 1974). While the authors comment upon the possibility and observational implications of this star being rotationally flattened in their first paper, they did not explicitly solve for this possibility, due to insufficient data to constrain an oblate model (Davis 2000). As such, Jordahl (1972) examines the Intensity Interferometer results in the context of of apparent disk brightness distribution resulting from stellar rotation theory, although this is done from the perspective of its effects upon the average angular diameter.

Observations of the overall diameter and projected shape of Altair upon the sky were taken using near-infrared, long-baseline interferometric measurements from PTI. PTI is an $85 \& 110 \mathrm{~m} \mathrm{H}$ - \& K-band $(1.6 \mu \mathrm{m} \& 2.2 \mu \mathrm{m})$ interferometer located at Palomar Observatory and is described in detail in Colavita et al. (1999). PTI has a minimum K-band fringe spacing of $\approx 4.3$ mas at the sky position of Altair, making this particular object readily resolvable.

\section{Observations of Altair}

Observations of Altair by PTI resulted in the rather surprising initial result that the apparent size of the star was dependent upon which interferometer baseline is used. The north-south baseline of PTI indicated the gross size of the star was 3.13 mas, while the north-west baseline indicated a somewhat larger size of 3.40 mas, as seen in Figure 1. The two baselines are rotated $50^{\circ}$ from each other, and their resultant measures across the stellar disk reflect a similar rotation relative to one another. Our surprise quickly evolved into suspicion of an instrumental 


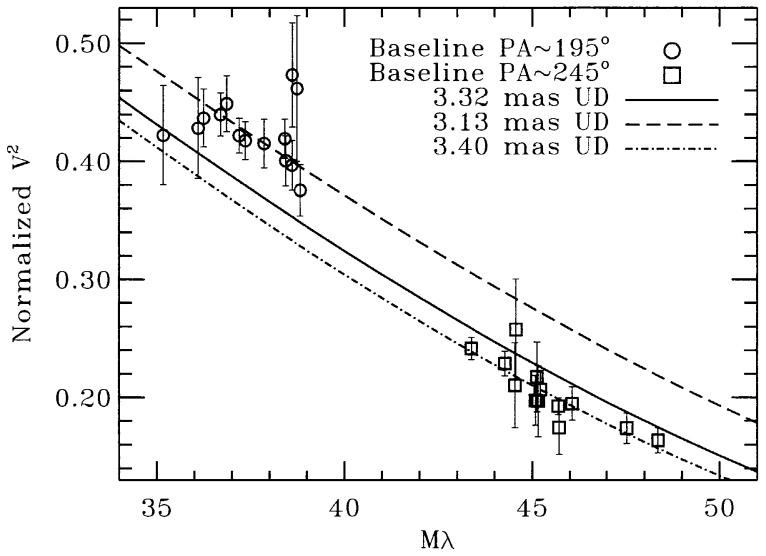

Figure 1. Visibility data for Altair. The visibility points at $\approx 37 \mathrm{M} \lambda$ correspond to a baseline projection angle of $\approx 195^{\circ}$ with a $3.137 \pm 0.025$ mas uniform disk angular size, and the visibility points at $\approx 45 \mathrm{M} \lambda$ correspond to a baseline projection angle of $\approx 245^{\circ}$ with a $3.400 \pm 0.018$ mas angular size. These two baseline projections result from the North-South and North-West baselines of PTI. A single 3.22 mas disk fit to all of the points clearly is inadequate in fully characterizing the data.

artifact, which was mitigated by a lack of such an effect in contemporaneous data taken on Vega (Ciardi et al. 2001, van Belle et al. 2001).

The key to understanding the peculiar diameter results for Altair lies in its rapid rotation. Different values for $v \sin i$ for the star have been derived from spectral line broadening profiles, depending upon the spectral line used: Carpenter et al. (1984) derive $190 \pm 38 \mathrm{~km} \mathrm{~s}^{-1}$ from IUE UV data; Freire Ferrero et al. (1978) derive $220 \mathrm{~km} \mathrm{~s}^{-1}$ from the visible Ca II lines; Stoeckley (1968) derives $250 \pm 10 \mathrm{~km} \mathrm{~s}^{-1}$ by observing visible $\mathrm{Mg} \mathrm{I}$ and $\mathrm{Ca}$ II lines. The raw data and additional visualizations of our Altair data are presented in our formal writeup (van Belle et al. 2001). Further details on the data collection and reduction process, and exclusion of competing hypotheses are also found in the manuscript.

In contrast to these values of $v \sin i$, the observed rotational velocity of Vega is roughly a factor of ten lower, at $<20 \mathrm{~km} \mathrm{~s}^{-1}$ (Freire Ferrero et al. 1983), which is consistent with its apparent lack of oblateness. While Vega has been reported to be a rapid rotator, this inference has been made in conjunction with the deduction that it is very nearly pole-on (Gulliver et al. 1994) with $i=5-6^{\circ}$, an orientation which would present the star to the interferometer as nearly circular upon the sky. As discussed in Ciardi et al. (2001) and van Belle et al. (2001), our data from PTI indicating circular symmetry for the disk of Vega are consistent with this conclusion.

We may investigate the effect of rotational distortion upon the photospheric appearance of a star. The force of centripetal acceleration at the equator, resulting from the rotation, offsets the effect of gravitation owing to the mass of the star. Under the conditions of hydrostatic equilibrium, uniform rotation, and a point mass gravitational potential, we may derive the equatorial rotational 
velocity, assuming we view the star at an inclination angle $i$. As developed in the work by Collins $(1963,1965)$ and presented in Jordahl (1972), we utilized an expression for the colatitude-dependent stellar radius at a rotation speed $u$ :

$$
r(\theta, u)=\frac{3}{u \sin \theta} \cos \left[\frac{\cos ^{-1}(-u \sin \theta)+4 \pi}{3}\right]
$$

As is appropriate in utilizing their mathematical constructs, we also adopted the coordinate system that is graphically illustrated in Collins (1965) and Jordahl (1972). Clearly there are more recent and/or more complicated models than this simple Roche model, but for the purposes of this analysis, we shall consider it sufficient. For the following computations, we used values of $1.8 \mathrm{M}_{\odot}$ for the mass of Altair (Malagnini \& Morossi 1990), and a parallax of $\pi=194.45 \pm 0.94$ mas (Perryman et al. 1997). Such a Roche model is applicable for a rigidly rotating star, which is consistent with a fully radiative stellar atmosphere. Fortunately, for the case of A-type main sequence star Altair, this is a reasonable expectation.

To interpret our radius data, we began by constructing models of Altair based upon rotation $u$ and polar radius $R_{p}(\omega)$, which are sufficient to map the entire surface as a function of stellar colatitude and longitude. Model surfaces were constructed for the full star at intervals of $0.5^{\circ}$ in both colatitude and longitude across the whole volume. These models were then mapped onto the sky and matched to the observational data through the use of two additional angular parameters, inclination $i$ and on-sky rotational orientation $\alpha$ at steps of $0.1 \mathrm{deg}$ along the entire circumference of the stellar limb.

Thus, for a given set of four randomized free parameters $\left\{u, R_{p}(\omega), i, \alpha\right\}$, a 300,000 point volume surface was generated, projected upon the sky, fit to the angular diameter data, and a $\chi^{2} / \mathrm{DOF}$ calculated. A multi-dimensional optimization code was then utilized to derive the best $\left\{u, R_{p}(\omega), i, \alpha\right\}$ solution from the random starting point, a process that took typically 500 iterations. (Press et al. 1992) An exhaustive search of the rotating star parameter space was used to explore the $\chi^{2} /$ DOF space. Furthermore, a static grid of $\{u, i\}$ values was explored for optimal $\left\{R_{p}(\omega), \alpha\right\}$ values to ensure that no local minima were trapping the optimization code. The grid consisted of 1,000 points spread uniformly over the space enclosed by $u=[0: 1], i=[0: 90]$ and was run multiple times with random $\left\{R_{p}(\omega), \alpha\right\}$ seed values, to ensure full mapping of the resultant $\{u, i\} \chi^{2} / \mathrm{DOF}$ surface. Trial runs of the $\chi^{2} / \mathrm{DOF}$ minimization technique using artificial data sets from synthetic stars were able to fully recover the original four parameter characterization for the original synthetic star. The model data sets covered a wider range of position angles, from $5^{\circ}$ to $175^{\circ}$ in $5^{\circ}$ steps, but with angular size errors comparable to the Altair dataset, which on average are $2.3 \%$ per measurement.

As seen in Figure 2, there is a noticable trough in the $\chi^{2} /$ DOF surface obtained via this technique, running from $u=0.77$ at $i=90^{\circ}$ (corresponding to viewing the star equator-on) to $u=1.00$ at $i=31.9^{\circ}$. Any inclination less than $31.9^{\circ}$ is physically inconsistent with our observed data. Unfortunately, there is no global minima that is differentiated from the other best fits in a statistically significant manner. Instead, we fit for the trough in $\{u, i\} \chi^{2} / \mathrm{DOF}$ space, mapping the family of models enclosed by +1 of $\chi^{2} / \mathrm{DOF}$ that describe 


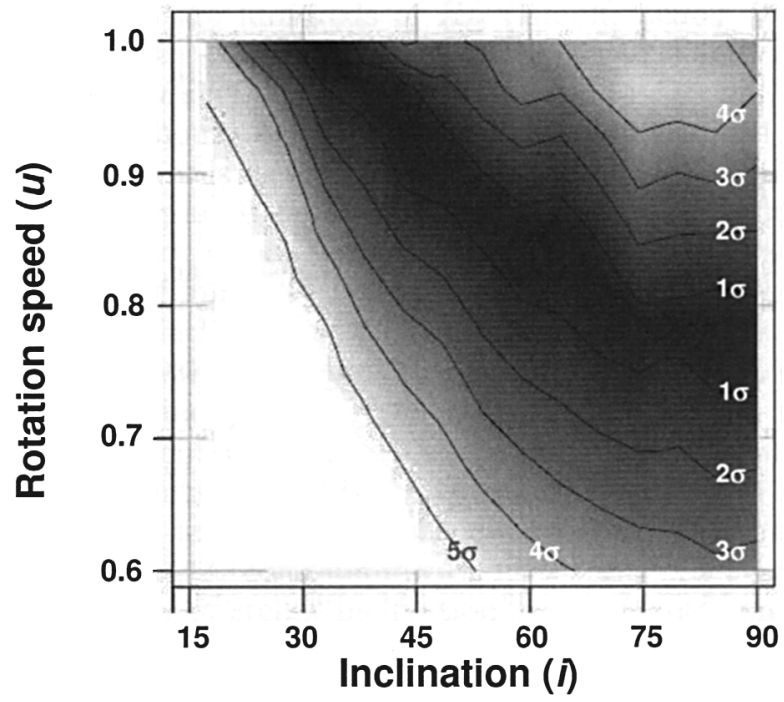

Figure 2. $\quad \chi^{2} /$ DOF surface for Altair as a function of rotation $u$ and inclination $i$.

the dependency of rotation $u$ upon inclination $i$ :

$$
u=4.961 \times 10^{-5}(90-i)^{2}+1.116 \times 10^{-3}(90-i)+0.762
$$

From each member of this family of models, derived values for angular velocity $\omega$, equatorial radius $R_{e}(\omega)$, equatorial velocity $v_{e}$, and apparent rotation velocity $v \sin i$ may be derived. An interesting aspect of this family of models is that they present uniform values for $v \sin i, R_{e}(\omega)$ and $\alpha$. Taking the ensemble of solutions found in the trough and averaging the result, we find that $v \sin i=$ $210 \pm 13 \mathrm{~km} / \mathrm{s}$. The preferred values for $R_{e}(\omega)$ and $\alpha$ are $1.8868 \pm 0.0066 R_{\odot}$ and $-21.6 \pm 6.2^{\circ}$ east of north for the pole, respectively. A illustration of a $\{u=$ $\left.0.82, i=70^{\circ}\right\}$ potential solution for our data is given in Figure 3. Examining predicted equatorial radii from the family of models, we are able to establish a critical rotation velocity of

$$
v_{\text {crit }}=\omega_{c} R_{e}\left(\omega_{c}\right)=\sqrt{\frac{G M}{R_{e}\left(\omega_{c}\right)^{3}}} R_{e}\left(\omega_{c}\right)=426 \pm 12 \mathrm{~km} / \mathrm{s},
$$

given our constant equatorial radius and assuming a $10 \%$ error on Altair's mass. This value is consistent with the value of $430 \mathrm{~km} / \mathrm{s}$ from Gray (1976).

An improvement to this technique would be to incorporate latitude dependent limb- and gravity-darkening, derived from a latitude-dependent temperature profile and projected onto the sky, resulting in a relationship between baseline projection angle and the limb darkening parameter $\mu$. Such an effort, however, is beyond the scope of this initial investigation into the observational appearance of rotational distorted photospheres. A variant of this approach has already been employed in the visible by Jordahl (1972), using the average angular size data of Hanbury Brown et al. (1974). In the near-infrared we expect 


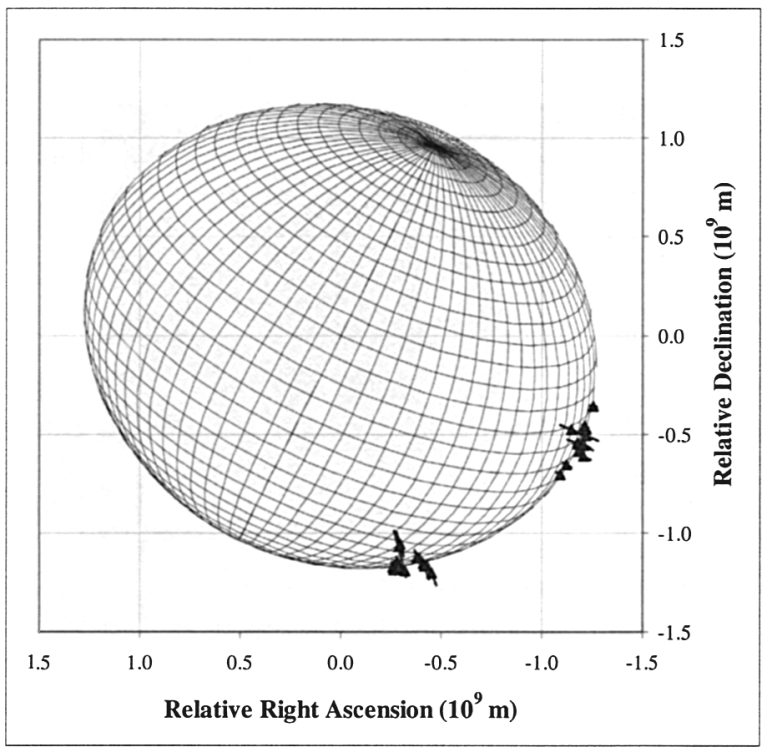

Figure 3. Example 3D model of Altair projected onto the sky $(u=0.82, i=$ $70)$, showing the fit of the PTI data to the limb of the stellar photosphere. Units are in meters at the distance of Altair.

this to be a much smaller effect than in the visible, at the $\approx 1 \%$ level, which is at a level much smaller than our typical angular size errors (discussed in much greater detail in van Belle et al. 2001). A potential next step in the development of this technique would be to combine multi-wavelength observations, potentially from multiple interferometers, to fit a ensemble of stellar models that features latitude-dependent temperatures.

Our derived value for $v \sin i$ is in good agreement with spectroscopically determined values, as presented in Section 3.5. The dominant source of error in our technique is primarily located in the angular size data, and secondarily in the mass estimate. The linear sizes are well constrained by the Hipparcos parallax, which has only a $0.4 \%$ quoted error, and dominated by the angular size error estimates of $\approx 2.3 \%$.

Conversely, we may take a measured value for $v \sin i$ in conjunction with our values for stellar radius and attempt to infer the mass of the star. Unfortunately, this approach is unduly sensitive to errors in both the rotational velocity and measures of the stellar shape. For example, if we were to take $v \sin i$ for Altair to be $220 \mathrm{~km} \mathrm{sec}^{-1}$ with a $10 \%$ error (cf. Freire Ferrero et al. 1995 and references therein), we measure the mass of this A7IV-V star to be $M=1.74 \pm 0.49 \mathrm{M}_{\odot}$. Substantial improvement in this measurement will be challenging: $1 \%$ values for polar, equatorial radii and $v \sin i$ still return only an $11 \%$ mass. A $1 \%$ mass from this technique requires $0.1 \%$ values for radii and rotational velocity, which appears currently highly challenging for both interferometry and spectroscopy.

Our measurements represent the first ever direct asymmetry observations for a main sequence star. We emphasize here that all other stars with reported asymmetries are either Mira variables or supergiant stars (cf. Lattanzi et al. 1997, Monnier et al. 1999). Previous work indicates that the radii of the Mira 
and supergiant stars are hundreds of $R_{\odot}$ (Haniff, Scholz \& Tuthill 1995, van Belle et al. 1996, van Belle et al. 1999); Equation 4 indicates a carbon star such as V Hya with $v \sin i \approx 14$ (Barnbaum et al. 1995) and $R \approx 400 R_{\odot}$ (van Belle et al. 1997) should exhibit an oblateness of $a / b \approx 1.11$. While this is remarkable in itself, it is perhaps an unsurprising expectation for these stars with extended atmospheres; in contrast to that is our finding of asymmetry for the relatively compact atmosphere of Altair. We are not aware of any other direct observations of luminosity class $\mathrm{V}$ or IV-V stars that indicate convincing departure from circularly-symmetric brightness distributions.

It it worth re-emphasizing that this technique can provide all four parameters $\left\{u, R_{p}(\omega), i, \alpha\right\}$, independently of each other. Although our existing Altair dataset was insufficient to provide more than the apparent rotation velocity $v \sin i$, our experiments during this investigation with the Monte Carlo simulations of synthetic stars with known deformation parameters indicate that sufficiently rich datasets, consistent with near-term observational capabilities, will allow for recovery of stellar inclination and true rotation velocity.

\section{Future Directions}

Despite the optimistic prognostications in van Belle et al. (2001) of an up and coming cottage industry of rapid rotator observations in optical interferometry, a single detection (and as of yet unconfirmed) does not constitute a substantive impact, aside from being a conceptual pathfinder. Our program of followup PTI observations on $\alpha$ Leo has been to date unsuccessful in producing meaningful results, primarily due to the small relative apparent size of this star (approximately a third that of Altair), and it appears that Altair may be the only star for which a measure of its oblateness may be obtained with PTI. However, this does not mean that this technique will dead end with this one detection of a rotationally distorted star.

In Table 1, a list of 67 prospective targets is given, along with an interferometer capable of observing the target. Target coordinates, visual magnitudes, and spectral types are taken from the Hipparcos catalog (Perryman et al. 1997); all targets were vetted for any sort of multiplicity flag in the Hipparcos catalog, which is indicative of binarity - a problem for interferometric observations of single stars. K magnitudes were taken from the 2MASS (Skrutskie et al. 2000) or Gezari et al. (1999) catalogs where available, although in a few cases these magnitudes were estimated. Apparent rotation velocities were taken from Glebocki, Gnacinski, \& Stawikowski (2000), and gross apparent angular size was estimated from $V$ and $K$ magnitudes, using the estimator found in van Belle (1999). Finally, a rough estimate of oblateness $R_{b} / R_{a}$ was calculated, based upon the predicted linear radius and mass as a function of spectral type, and

$$
v \sin i \approx \sqrt{\frac{2 G M}{R_{b}}\left(1-\frac{R_{b}}{R_{a}}\right)},
$$

an approximation that is a simplification of an expression describing self-gravitating rotationally distorted gaseous masses (Baron et al. 1989). For Altair, which has a measured ratio of $R_{b} / R_{a} \approx 1.14$, this expression predicts a value of 1.16 . 
Table 1. Catalog of prospective rotationally distorted stars, as discussed in $\S 3$.

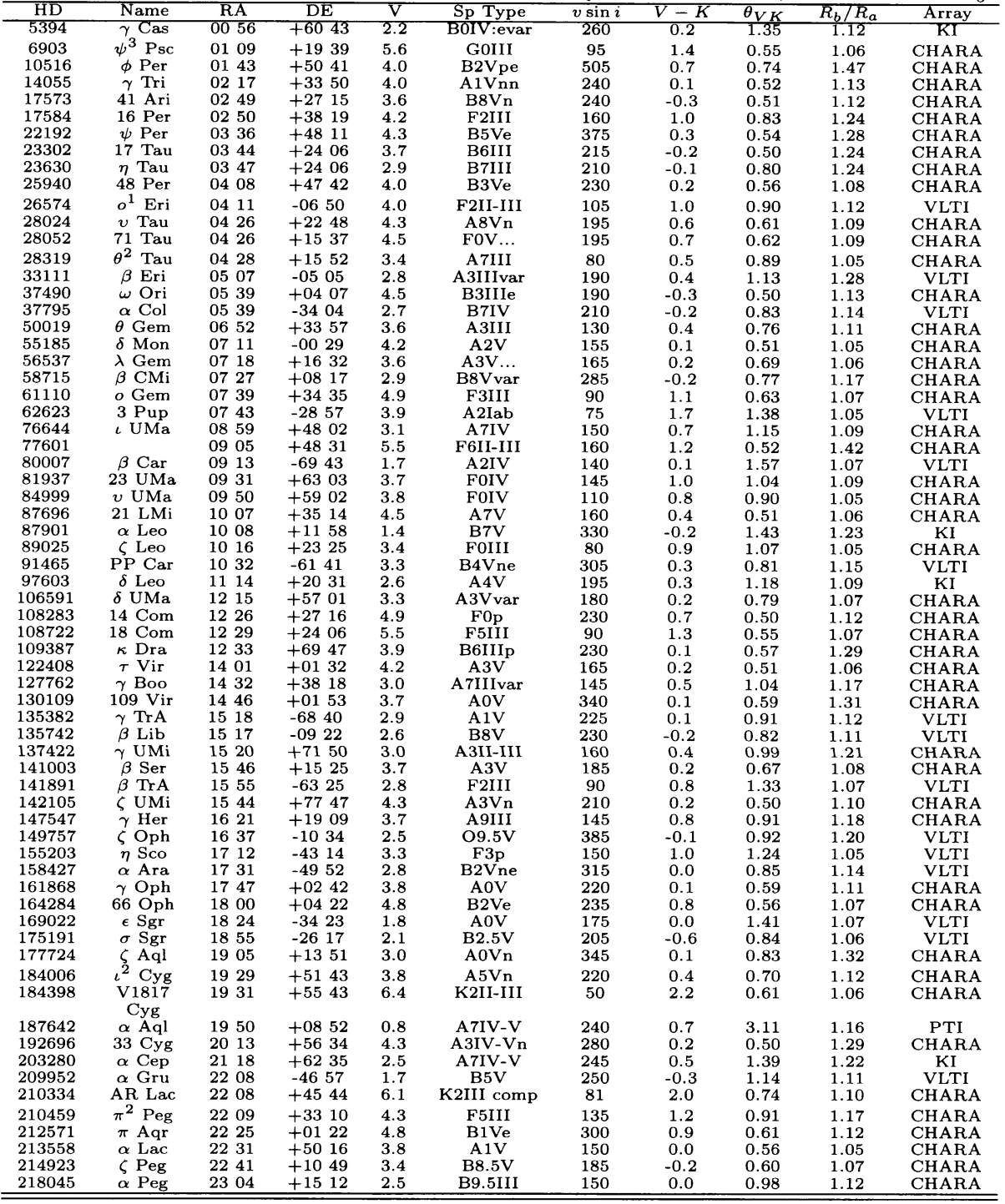

Table 2. Summary of targets by interferometer, as discussed in $\S 3$.

\begin{tabular}{ccc}
\hline Array & $>10 \%$ oblate & $5 \%-10 \%$ oblate \\
\hline CHARA & 26 & 21 \\
KI & 3 & 1 \\
NPOI & 30 & 22 \\
PTI & 1 & 0 \\
VLTI & 10 & 5 \\
\hline \hline
\end{tabular}


Three new arrays were considered in compiling the list: the Georgia State University Center for High Angular Resolution Array (CHARA), the Keck Interferometer (KI), and the Very Large Telescope Interferometer (VLTI). These arrays will offer sensitivity in the near infrared similar to, or greater than, that of PTI, with increases in spatial resolution afforded by longer baselines, and required for increases in potential sample sizes of these targets. Also worth considering is the Navy Prototype Interferometer (NPOI), a reconfigurable array that has the potential to observe all of the northern hemisphere targets in the visible. NPOI is of particular interest for these observations, in that its shorter operational wavelength opens a sensitive window for probing not just atmospheric oblateness but latitude dependencies upon surface temperature as well. Combining observations from NPOI with near-infrared observations from another instrument should prove to be especially useful for reconstructing meaningful stellar surface maps. Targets were chosen for the arrays based upon which stellar hemisphere they resided in, and the expected angular size of the target resulting in a visibility between 0.2 and 0.5 , a range of values where interferometers do their most sensitive measures of size.

Confirming the existence of a this large target catalog, accessible to instruments that are not merely planned but in initial stages of operation, presages the day when statistically significant quantities of these objects have been observed at microarcsecond resolutions. A summary of the target quantities by instrument can be found in Table 2.

We are in the process of encouraging others in the interferometric community to pursue similar investigations with these instruments, and are furthering our own investigations of rotating stars as well. Recently one of the authors (GvB) was awarded VLTI time to attempt an initial southern hemisphere program, using the temporary siderostat feeds in place at the site. It is our anticipation that additional observations of rotational oblateness will trickle in only slowly over the next 12 to 24 months prior to facility checkouts, after which there should be a steady stream of new information from this new frontier in the field. Our experience with bringing interferometric data to other areas of astronomy indicates that this complimentary set of fundamental observations will refine and further our understanding of stellar structure.

\section{References}

Allen, C.W., 1973, Astrophysical Quantites, London: University of London, Athlone Press, 3rd ed

Barnbaum, C., Morris, M., Kahane, C., 1995, ApJ 450, 862

Baron, R.L., French, R.G., Elliot, J.L., 1989, Icarus, 78, 119

Carpenter, K.G., Slettebak, A., Sonneborn, G., 1984, ApJ 286, 741

Ciardi, D.R., van Belle, G.T., Thompson, R.R., Akeson, R.L., Lada, E.A., Howell, S.B., 2001, ApJ 559, 1147

Colavita, M.M., et al., 1999, ApJ 510, 505

Collins, G.W., II, 1963, ApJ 138, 1134

Collins, G.W., II, 1965, ApJ 142, 265

Davis, J., 2000, private communication

Freire Ferrero, R., Praderie, F., Czarny, J., Felenbok, P., 1978, A\&A 61, 785 
Freire Ferrero, R., Gouttebroze, P., Catalano, S., Marilli, E., Bruhweiler, F., Kondo, Y., van der Hucht, K., Talavera, A., 1983, ApJ 439, 1011

Gezari, D.Y., Pitts, P.S., Schmitz, M., 1999, Catalog of Infrared Observations, Edition 5

Glebocki, R., Gnacinski, P., \& Stawikowski, A., 2000, AcA 50, 509

Gouttebroze, P., Freire Ferrero, R., Marilli, E., Catalano, S., 1999, A\&A 348, 198

Gray, D.F., 1976, The Observation and Analysis of Stellar Photospheres, New York, Wiley-Interscience

Gulliver, A.F., Hill, G., Adelman, S.J., 1994, ApJL, 429, L81

Hanbury Brown, R., Davis, J., Allen, L.R., Rome, J.M., 1967, MNRAS 137, 393

Hanbury Brown, R., Davis, J., Allen, L.R, 1974, MNRAS 167, 121

Haniff, C.A., Scholz, M., Tuthill, P.G., 1995, MNRAS 276, 640

Johnson, H.L., Morgan, W.W., 1953, ApJ 117, 313

Jordahl, P.R., 1972, Ph.D. thesis, University of Texas at Austin.

Lattanzi, M.G., Munari, U., Whitelock, P.A., Feast, M.W., 1997, ApJ 485, 328

Malagnini, M.L., Morossi, C., 1990, A\&AS, 85, 1015

Monnier, J.D., Tuthill, P.G., Danchi, W.C., 1999, ApJ 525, L97

Perryman, M.A.C., et al., 1997, A\&A 323, 49

Press, W.H., Teukolsky, S.A., Vetterling, W.T., Flannery, B.P., 1992, Numerical Receipes in C, Port Chester, Cambridge University Press

Schlesinger, F., 1909, Publ. Allegheny Observatory 1, 134

Schlesinger, F., 1911, MNRAS 71, 719

Skrutskie, M.F., et al., 2000, Proc. Workshop "The Impact of Large Scale Near-IR Sky Surveys", 25

Stoeckley, T.R., 1968, MNRAS 140, 121

van Belle, G.T., Dyck, H.M., Benson, J.A., Lacasse, M.G., 1996, AJ 112, 2147

van Belle, G.T., Dyck, H.M., Thompson, R.R., Benson, J.A., Kannappan, S.J., 1997, AJ 114, 2150

van Belle, G.T., 1999, PASP, 111, 1515

van Belle, G.T., Lane, B.F., Thompson, R.R., The PTI Collaboration, 1999, AJ 117, 521

van Belle, G.T., Ciardi, D.R., Thompson, R.R., Akeson, R.L., Lada, E.A., 2001, ApJ 559,1155 